\title{
PRIAPISMO COMO PRESENTACIÓN INICIAL DE LEUCEMIA MIELOIDE CRÓNICA
}

\author{
M. ALluÉ LÓPEZ, Á. GARCÍA DE JALÓN MARTÍNEZ, D. PASCUAL REGUEIRO, \\ E. MALLÉN MATEO, A. VILLANUEVA BENEDICTO, L.Á. RIOJA SANZ
}

Servicio de Urología. Hospital Universitario Miguel Servet. Zaragoza.

Actas Urol Esp. 28 (5): 387-389, 2004

\section{RESUMEN}

PRIAPISMO COMO PRESENTACIÓN INICIAL DE LEUCEMIA MIELOIDE CRÓNICA

Se presenta el caso de un paciente de 29 años de edad que presentó cuatro episodios de priapismo en los últimos tres meses, sin asociarse a ningún otro síntoma y sin traumatismo previo.

Tras la realización de un hemograma se demuestra que la aparición de estos repetidos episodios de priapismo se debe una leucemia mieloide crónica.

PALABRAS CLAVE: Priapismo. Leucemia mieloide crónica.

\section{ABSTRACT \\ PRIAPISM AS AN INICIAL PRESENTATION OF CHRONIC MYELOID LEUKAEMIA}

We report the case of a 29 years old patient who presented, in the last three moths, four episodes of priapism without any other symptoms and any previous traumatism.

An hemogram reveled that the repeted episodes of priapism were caused by a chronic myeloid leukaemia.

KEY WORDS: Priapism. Chronic myeloid leukaemia.

$\mathrm{E}$ 1 priapismo es una erección peneana prolongada y dolorosa, no asociada con un estímulo sexual.

Existen dos tipos de priapismo: de bajo flujo (veno-oclusivo o isquémico) y de alto flujo (arterial o no isquémico). El segundo suele tener un origen traumático, mientras que el primero puede ser debido a múltiples etiologías, entre las que destacan: causas hematológicas (anemia de células falciformes, leucemias, alteraciones de la agregación plaquetaria,...), neoplasias locales, infecciones, fármacos, inyecciones intracavernosas para el tratamiento de la disfunción eréctil. En el 30-50\% de los casos no se identifica la causa del priapismo ${ }^{1}$.
Las opciones terapéuticas y la urgencia con que deben ser aplicadas, difieren en cada tipo de priapismo por lo que es importante diferenciarlos de antemano.

El tratamiento de priapismo de bajo flujo debe ser inmediato para evitar el daño de los cuerpos cavernosos y la pérdida de función sexual, mientras que si se trata de priapismo de alto flujo el tratamiento puede ser diferido ya que no existe riesgo de lesionar la futura capacidad eréctil del pene $^{2,3}$.

\section{CASO CLÍNICO}

Paciente de 29 años de edad que acude al Servicio de Urgencias de nuestro Hospital ante la 
presencia de una erección levemente dolorosa de diez horas de evolución. El paciente refiere haber presentado cuatro episodios similares en los últimos tres meses pero en todos ellos la erección había cedido como máximo en tres o cuatro horas.

El paciente niega el antecedente de traumatismo peneano, el uso de drogas $\mathrm{u}$ otros fármacos incluidas inyecciones intracavernosas. Así mismo no presenta ningún otro síntoma acompañante como astenia, anorexia o fiebre.

Se realiza una gasometría de la sangre extraída de los cuerpos cavernosos obteniendo resultados analíticos de sangre venosa (pCO2 163,1 mmHg, HCO3 13,2 mEq/1 plasma).

Ante este priapismo de prolongada duración y la ausencia de indicios que nos hagan pensar en un priapismo de alto flujo, procedemos a intentar revertir la erección con la mayor brevedad posible, con el fin de calmar el dolor y evitar un daño irreversible en los cuerpos cavernosos.

Se punciona con un catéter intravenoso de 19 G en ambos cuerpos cavernosos, aspirando sangre en repetidas ocasiones y se deja que ésta gotee. Tras este primer paso no se obtiene disminución de la erección, por lo que se prepara una solución de fenilefrina (agonista alfaadrenérgico) en suero salino a una concentración de $0,1 \mathrm{mg} / \mathrm{ml}$. Se inyecta $2 \mathrm{ml}$ de la solución en un cuerpo cavernoso, comprimiendo posteriormente. Se repite nuevamente esta maniobra en el cuerpo cavernoso contralateral. Ante la ausencia de respuesta, se realiza una nueva solución de fenilefrina, mezclando $10 \mathrm{mg}$ de fenilefrina en 11 de suero salino y se van introduciendo $20-30 \mathrm{ml}$ de esta solución en cada uno de los cuerpos cavernosos para lavarlos. Tras la repetición en varias ocasiones de esta última maniobra, se consigue obtener finalmente flaccidez peneana.

Durante su permanencia en urgencias y tras lograr flaccidez, se extraen analíticas sanguíneas que revelan una marcada elevación de las cifras de leucocitos $\left(414 \times 10^{9} / \mathrm{L}\right)$ y plaquetas $(1100 \mathrm{x}$ $10^{9} / \mathrm{L}$ ) en sangre.

Ante la sospecha de un síndrome mieloproliferativo se consulta con el Servicio de Hematología, que diagnostica leucemia mieloide crónica (Figs. 1 y 2).

Posteriormente el paciente es sometido a un trasplante de médula ósea alogénico de donante familiar con HLA idéntico. La evolución de su enfermedad hematológica es favorable quedando el cuadro de priapismo totalmente resuelto al tratar la leucemia mieloide crónica y sin alteración en la potencia sexual.

\section{DISCUSIÓN}

La causa más frecuente de priapismo es el tratamiento de la disfunción eréctil con inyecciones intracavernosas.
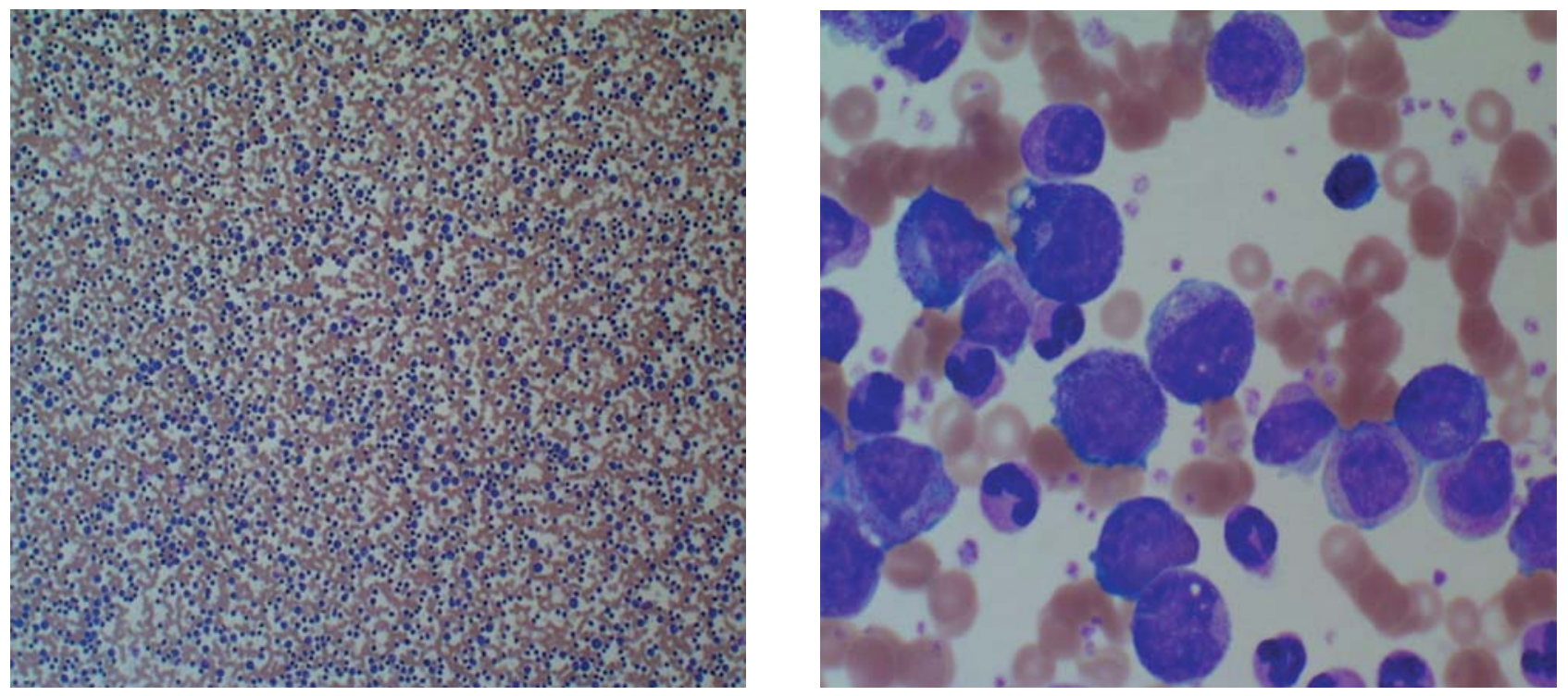

FIGURA 1. a) Extensión sanguínea a 10 aumentos de magnificación original teñida con Giemsa, que muestra la excesiva celularidad de la serie granulocítica; b) Tinción de Giemsa con aceite de inmersión a 100 aumentos. 


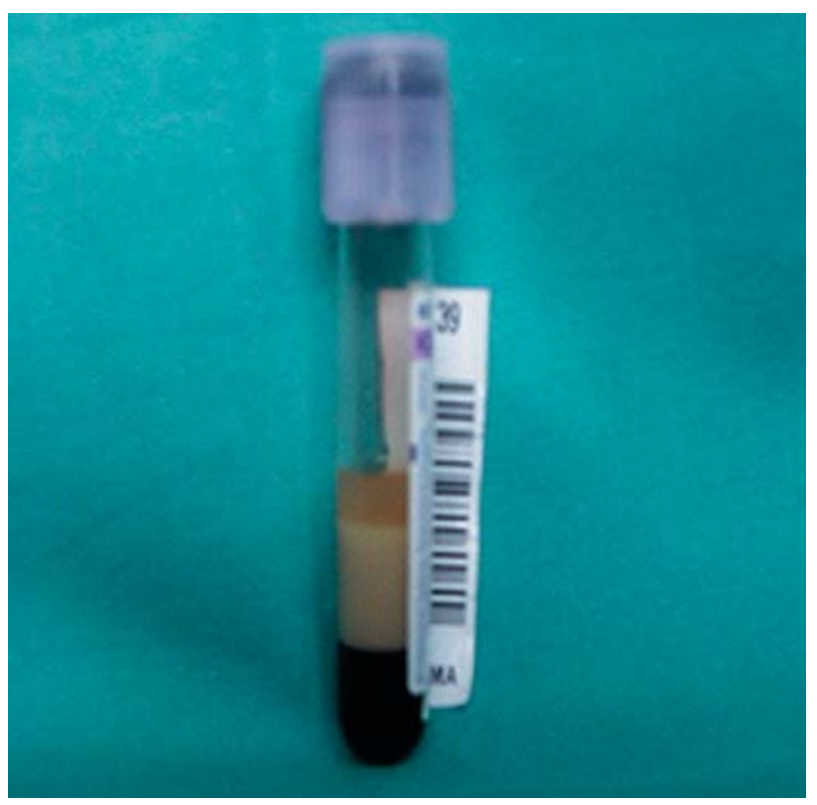

FIGURA 2. La importante leucocitosis se ve claramente reflejada en este tubo de extracción por la gran cantidad de células de la serie blanca.

Sólo en el $1 \%$ de los casos de priapismo, la etiología es una leucemia. Además, el priapismo asociado a enfermedades proliferativas suele ser debido principalmente a leucemia mieloide crónica, aunque también puede darse en leucemia linfoide crónica. Por otro lado, aunque la leucemia mieloide crónica representa el $5 \%$ de las leucemias, es responsable del $50 \%$ de los casos de priapismo leucémico ${ }^{4}$.

La etiología del priapismo leucémico se desconoce, pero principalmente se barajan la hiperviscosidad, la aglutinación sanguínea por la leucocitosis, y la obstrucción mecánica de las venas y nervios abdominales por esplenomegalia 4 .

Las medidas terapéuticas de los dos tipos de priapismo son muy distintas por lo que primero debería realizarse una gasometría cavernosa y un eco-doppler (permitirá la visualización de la fístula en caso de priapismo de alto flujo) para diferenciarlos.

Si tras los pasos dados en este paciente para el tratamiento del cuadro agudo de priapismo de bajo flujo no se hubiera obtenido flaccidez, se pasaria a realizar un shunt caverno-esponjoso trasbalánico, y si esta medida fuera insuficiente, la última opción sería la confección de un shunt caverno-esponjoso quirúrgico ${ }^{2,3}$.

En el priapismo de alto flujo, el tratamiento sería la realización de una embolización selectiva y si ésta fallase, una ligadura quirúrgica selecti$\mathrm{va}^{2,3}$.

A diferencia del priapismo de bajo flujo, el de alto flujo no es una urgencia, ya que existe una buena perfusión sanguínea peneana.

El tratamiento del priapismo debe ir dirigido a tratar la causa primaria, si es que se identifica, con el fin de obtener flaccidez con la mayor brevedad posible, calmar el dolor y prevenir el daño permanente de los cuerpos cavernosos, lo que conduciría a una pérdida de la potencia sexual ${ }^{5,6}$. En el caso presentado, la etiología del priapismo es la leucemia mieloide crónica, por lo que su curación impide la nueva aparición de episodios de erección prolongada.

\section{REFERENCIAS}

1. LUE TF.: Physiology of erection and pathophysiology of impotency. Campbell's Urology. 6ª , 1992: 709727.

2. BYRON E, LÓPEZ DE MESA L, JAVIER VALERO J, MALO RODRÍGUEZ G.: Priapismo en niños (reporte de un caso). Hospital de la Misericordia. Unidad de Urologia Pediátrica. Santa Fe de Bogotá, Colombia. Publicación de la Sociedad Colombiana de Urología, mayo 1999; 8 (1).

3. NURZIA MICHAEL J, STANLEY ZASLAU MD, VAPNEK JONATHAN M.: Priapism as an inicial presentation of chronic myelogenous leukemia. Departament of Urology. Mount Sinai School of Medicine. New York. Hospital Physician 1999 november.

4. BENSON GS.: Priapismo. AUA Update Series 1996; 15. Lesson.

5. ROJAS B, CABRERA ME, KLIWADENCO W, AVILÉS JJ.: Priapism in a patient with chronic myeloid leukemia. Rev Med Chil 1998 aug; 126 (8): 978980.

6. GHALAUT PS, KALRA GS, GUPTA S.: Priapism -a rare presentation in chronic myeloid leukaemia. $J$ Assos Physicians India 1995 apr; 43 (4): 297.

Dra. M. Allué López

C/ Asalto, $67-5^{\circ} \mathrm{D}$

50002 Zaragoza

(Trabajo recibido el 21 marzo de 2003) 